\title{
EVOLUÇÃO DA INFESTAÇÃO POR Sirex noctilio EM FUNÇÃO DA DISTRIBUIÇÃO DIAMÉTRICA EM PLANTIOS DE Pinus taeda
}

\author{
Débora Cristina Machado Gaiad ${ }^{1}$ \\ Afonso Figueiredo Filho ${ }^{2}$ \\ Edilson Batista de Oliveira ${ }^{3}$ \\ Susete do Rocio Chiarello Penteado ${ }^{4}$
}

\begin{abstract}
RESUMO
Este trabalho teve como objetivo analisar a evolução do ataque de Sirex noctilio (vespa-da-madeira), em função da distribuição diamétrica em plantios de Pinus taeda desbastados e não desbastados. Os dados utilizados no estudo foram provenientes de um experimento com cinco tratamentos e cinco repetições, instalado em três áreas da empresa Klabin-Celucat no estado de Santa Catarina, em parceria com a Embrapa Florestas. Os tratamentos foram aplicados em sub-áreas de $3600 \mathrm{~m}^{2}$ com parcelas amostrais de $400 \mathrm{~m}^{2}(20 \times 20 \mathrm{~m})$ instaladas no centro de cada sub-área. $\mathrm{O}$ delineamento experimental usado foi blocos ao acaso. Foram testados os seguintes tratamentos: (a) T1 - testemunha (sem desbaste); (b) T2 - 25\% (4 linha) desbaste sistemático e $10 \%$ seletivo nas remanescentes; (c) T3 - 25\% sistemático e $20 \%$ seletivo; (d) T4 - 25\% sistemático e $30 \%$ seletivo; (e) T5 - 25\% sistemático e $40 \%$ seletivo. No desbaste seletivo foram retiradas, preferencialmente, as árvores atacadas, bifurcadas e dominadas. Foram coletadas informações sobre DAP (diâmetro à altura do peito), h (altura total) e condição fitossanitária (sadia ou atacada) das árvores de cada parcela, antes e depois da aplicação de cada tratamento a fim de relacionar a infestação da vespa-da-madeira com o vigor das árvores. Neste estudo concluiuse que: (a) o desbaste retarda o ritmo de ataque da vespa-da-madeira e pode ser utilizado como medida adicional de controle em um programa de manejo integrado de pragas; (b) o ataque da vespa-da-madeira inicia-se pelas árvores de menor diâmetro e atinge as de maior diâmetro com o aumento da infestação, ocorrendo um deslocamento ascendente no pico de ataque das classes de menor para as de maior diâmetro.
\end{abstract}

Palavras chave: estrutura horizontal, vespa-da-madeira, desbastes, ataque

\section{EVOLUTION OF Sirex noctilio INFESTATION IN REALTION TO DIAMETER DISTRIBUTION ON Pinus taeda}

\begin{abstract}
This paper aimed to analyze the evolution of Sirex noctilio (woodwasps) attack in relation to diameter distribution in thinned and unthinned Pinus taeda stands. The data were collected at Klabin-Celucat Company on a partnership with the National Center of Forest Research from Embrapa. The treatments were set up at $3600 \mathrm{~m}^{2}$ sub-plots with a $400 \mathrm{~m}^{2}(20 \times 20)$ sampling area at the center of each sub-plot. The experimental design was randomized blocks. The following treatments were tested: (a) T1 - control (no thinning); (b) T2 - 25\% systematic thinning followed by a $10 \%$ selective thinning; (c) T3 - 25\% systematic thinning followed by a $20 \%$ selective thinning; (d) T4 - 25\% systematic thinning followed by a 30\% selective thinning; T5 - 25\% systematic thinning followed by a $40 \%$ selective thinning. At the selective thinning were withdraw the attacked, forked and dominated trees. It were taken data on DBH (diameter at breast height), h (total height) and health condition (attacked or not attacked) of trees before and after the treatments implantation, aiming to establish relationship between the woodwasp infestation and tree vigor. It was concluded that: (a) thinning operations delay the rhythm of woodwasp attack and can be used as additional measure of control on an integrated pest management program; (b) the woodwasp attack starts by the trees with smaller diameters and reach the trees with bigger diameter as the infestation rises moving up the peak of attack from smaller classes of diameter towards the higher classes.
\end{abstract}

Key-words: horizontal structure, woodwasp, thinning, attack

\footnotetext{
${ }^{1}$ Engenheira Florestal, Mestre Ciências Florestais, gaiad@uol.com.br

${ }^{2}$ Engenheiro Florestal, Doutor Ciências Florestais, afig@floresta.ufpr.br, UFPR, Curitiba, PR / UNICENTRO, Irati, PR

${ }^{3}$ Engenheiro Florestal, Doutor Ciências Florestais, edilson@cnpf.embrapa.br, Embrapa Florestas, Colombo, PR

${ }^{4}$ Bióloga, Mestre Ciências Biológicas, susete@cnpf.embrapa.br, Embrapa Florestas, Colombo, PR
} 


\section{INTRODUÇÃO}

Grande parte dos povoamentos de Pinus spp implantados no Brasil na década de 60 , sofreram pouca ou nenhuma intervenção silvicultural. Isto associado ao fato destes plantios serem homogêneos e de altas densidades, ocasionou um declínio no vigor das árvores e, conseqüentemente, nas condições fitossanitárias do povoamento, tornando-os suscetíveis ao ataque de pragas.

Sirex noctilio (vespa-da-madeira) detectada em 1988 encontrou nestes povoamentos debilitados, as condições ideais para seu estabelecimento e dispersão, acarretando as maiores perdas registradas em plantios de Pinus até hoje no Brasil.

O manejo florestal é uma ferramenta importante nos programas de prevenção e controle de Sirex noctilio, pois atua diretamente sobre as condições fitossanitárias do povoamento. A realização de desbastes contribui para a melhoria das árvores remanescentes, pois promove seu crescimento individual e diminui a competição por luz, água e nutrientes, tornando-as mais vigorosas e resistentes ao ataque deste inseto.

Este trabalho teve como objetivo analisar a evolução do ataque de Sirex noctilio na distribuição diamétrica de plantios de Pinus taeda desbastados e não desbastados.

\section{REVISÃO BIBLIOGRÁFICA}

Insetos fazem parte de ecossistemas florestais e podem ter impactos econômicos e sociais negativos. Estes impactos adversos devem ser prevenidos ou mantidos a níveis toleráveis, por meios ecológicos que sejam compatíveis com os objetivos e práticas do manejo florestal (Waters e Stark, 1980). A suscetibilidade das árvores ao ataque de insetos, varia com a composição da floresta, idade, densidade, sítio e vigor. Grandes povoamentos homogêneos e eqüiâneos são mais suscetíveis ao ataque de pragas que povoamentos de idades ou espécies diferentes (Davis, 1966). Devido a grande proporção de árvores disponíveis ao ataque e ampla oferta de alimento, as monoculturas propiciam condições ótimas para o desenvolvimento do inseto (Alfaro, 1996).

Sirex noctilio foi detectado no Brasil em fevereiro de 1988, em povoamentos de
Pinus taeda de 17 anos de idade, densidade inicial de 2500 plantas/ha, não desbastados, sendo que o estresse causado pela competição favoreceu o desenvolvimento do inseto (Iede $e t$ al., 1988).

A ausência de desbaste leva ao estresse da floresta e à obtenção de matériaprima de baixa qualidade e menores bitolas, levando a uma menor remuneração do empreendimento e facilitando o aparecimento de pragas e/ou doenças (Scolforo e Maestri, 1997).

O desbaste contribui para melhorar a disposição das árvores remanescentes, proporcionando um maior espaço aéreo para o desenvolvimento das copas, uma maior superfície de produção fotossintética e, uma maior disponibilidade de água e nutrientes, para um menor número de indivíduos (Monteiro Alves, $1982^{5}$ citado por Mainardi et al., 1996).

A maior parte dos desbastes reduz as perdas ocasionadas por agentes daninhos, não somente pela prevenção, como também pelo aumento do vigor e resistência das árvores. Somente sob circunstâncias especiais, o desbaste aumenta a suscetibilidade ao ataque de insetos (Smith, 1986).

$\mathrm{O}$ monitoramento dos plantios e práticas silviculturais que incluam desbastes seletivos, com remoção de árvores danificadas, doentes e bifurcadas, são medidas preventivas importantes para o controle de insetos como a "vespa-da-madeira". As árvores que resistem ao ataque de $S$. noctilio são aquelas sem nenhum tipo de dano físico e que cresceram em condições adequadas (Neumann et al., 1987).

Estudos realizados na Austrália, em povoamentos de 17 anos de idade, infestados e não desbastados, indicaram que o nível de mortalidade de árvores atacadas por $S$. noctilio é inversamente proporcional aos seus diâmetros. Desta forma árvores com DAP $<23$ $\mathrm{cm}$ apresentaram um índice de mortalidade significativamente maior que às com DAP > $29 \mathrm{~cm}$. Árvores com DAP $>35 \mathrm{~cm}$ permaneceram livres de ataque e árvores

5 MONTEIRO ALVES, A.A. Técnicas de produção florestal: fundamentação, tipificação e métodos. Lisboa. Instituto Nacional de Investigação Científica. 1982. 331 p. 
bifurcadas foram mais suscetíveis (Neumann et al., 1987).

$\mathrm{O}$ ataque de $S$. noctilio na Nova Zelândia, Tasmânia e Continente Australiano, resultou na morte, principalmente, das árvores dominadas. $\mathrm{O}$ sombreamento pareceu ser a causa mais importante da morte de árvores, pois árvores sombreadas artificialmente morreram mais rapidamente que às não sombreadas (Coutts, 1968).

Devido ao fato de $S$. noctilio ser atraída por árvores estressadas ou recentemente mortas, a prevenção e o controle deste inseto através da utilização de práticas silviculturais, tem sido amplamente recomendada (Madden, 1975; Neumann, 1979; Taylor, 1981; Neumann et al., 1987).

\section{MATERIAIS E MÉTODOS}

O experimento foi instalado em 1992 em povoamentos de Pinus taeda da KLABIN CELLUCAT, em uma parceria com a EMBRAPA - Florestas, em três áreas com início de ataque da vespa-da-madeira no estado de Santa Catarina: a) Fazenda Cerro Pelado no município de São José do Cerrito com 13 anos de idade; b) Fazenda Goiabeira no município de Bocaina do Sul com 12 anos de idade e c) Fazenda Marco no município de Curitibanos, com 12 anos de idade.

Foram escolhidos povoamentos com densidade inicial de 2000 plantas/ha $(2,0 \mathrm{x}$ 2,5m), sem desbaste, utilizando-se o delineamento experimental de blocos ao acaso. Em cada fazenda foram instalados cinco blocos com cinco tratamentos. Os tratamentos testados foram os seguintes: (a) sem desbaste testemunha (T1); (b) os demais tratamentos foram desbastes sistemático e seletivo, onde o sistemático foi constante $\left(25 \%-4^{\mathrm{a}}\right.$ linha $)$ e o seletivo foi aumentando progressivamente em 10\% (T2); 20\% (T3); 30\% (T4) e 40\% (T5).

Os tratamentos foram aplicados no ano de 1993, em sub-áreas de $3600 \mathrm{~m}^{2}$ com parcelas amostrais de $400 \mathrm{~m}^{2}(20 \mathrm{x} \quad 20)$ instaladas no centro de cada sub-área. Cada bloco totalizava $18.000 \mathrm{~m}^{2}$, sendo que o número de árvores dentro de cada parcela variou de 64 a 94 . No desbaste seletivo, foram retiradas, preferencialmente, as árvores atacadas, bifurcadas e dominadas.
Foram coletadas informações do DAP (diâmetro à altura do peito), h (altura total) e condição fitossanitária (sadia ou atacada) das árvores de cada parcela, antes (1992 e 1993) e depois (1994, 1995, 1996 e 1999) da aplicação de cada tratamento, sendo que os dados de 1992 da Fazenda Goiabeira foram perdidos.

Para este trabalho, as árvores atacadas de cada parcela foram agrupadas em classes de DAP de $5 \mathrm{~cm}$ de amplitude nos diferentes anos de medição. Essa amplitude foi definida em função da distribuição dos dados de forma que representasse toda a variabilidade diamétrica dos tratamentos e das várias idades consideradas. $\mathrm{O}$ agrupamento dos dados teve como finalidade verificar a relação existente entre a infestação e o diâmetro de árvores atacadas, definindo as árvores preferidas pela vespa-da-madeira e o deslocamento da infestação pelas classes diamétricas.

\section{RESULTADOS E DISCUSSÃO}

Como a variável DAP foi medida de 1992 a 1999 foi possível verificar o comportamento do inseto, em relação às classes diamétricas.

O Tratamento T1 (sem desbaste) apresentou os menores valores diamétricos médios (aritméticos). Em relação aos outros tratamentos observou-se que conforme aumentam as intensidades do desbaste, aumentam os diâmetros médios, em uma relação diretamente proporcional (fig. $1 \mathrm{e}$ quadro 1).

Este resultado está de acordo com a literatura, uma vez que árvores, em povoamentos superestocados, crescem abaixo do permitido pelo ambiente (Mitchell et al., 1983). O desbaste melhora a condição das árvores remanescentes, acarretando em uma maior disponibilidade de água e nutrientes (Mainardi et al., 1996) e favorecendo o seu crescimento diamétrico (Smith, 1986).

Os resultados da análise dos diâmetros das árvores atacadas, em relação as classes diamétricas, são apresentados para as três fazendas, Cerro Pelado (fig. 2), Goiabeira (fig. 3) e Marco (fig. 4). Constatou-se que a quantidade de árvores infestadas é diferente nas várias classes diamétricas. $\mathrm{O}$ ataque iniciou 
pelas árvores de menor diâmetro e com o aumento da infestação, passou para as árvores maiores, ocorrendo um deslocamento ascendente no pico de ataque das classes de menor para as de maior diâmetro.

Observa-se (fig. 2, 3 e 4) uma maior incidência de ataque de $S$. noctilio no tratamento T1 em 1999 (19 anos para a Fazenda Cerro Pelado e 17 anos para as Fazendas Goiabeira e Marco). Os maiores e menores percentuais de árvores infestadas foram encontrados na Fazenda Goiabeira e Fazenda Marco, respectivamente. O tratamento T1 (sem desbaste) apresentou um nível de ataque superior aos tratamentos desbastados, deixando evidente que o desbaste contribui para o controle desta praga.

A maioria das árvores atacadas encontrava-se nas classes de DAP $\leq 25$, sendo detectadas poucas árvores atacadas com DAP $>35 \mathrm{~cm}$. Estes dados confirmam os resultados de MADDEN (1975) que destaca que o ataque de $S$. noctilio, em povoamentos de Pinus spp., inicia-se pelas árvores de menor diâmetro (dominadas), atingindo árvores codominantes e dominantes conforme o estabelecimento da praga se consolida. Resultados semelhantes foram obtidos por Neumman et al. (1987) que estudando povoamentos de $P$. radiata, não desbastados, com 17 anos, constataram que os maiores níveis de ataque se concentravam em árvores com DAP abaixo de $23 \mathrm{~cm}$. Árvores acima de $26 \mathrm{~cm}$ foram menos atacadas e, árvores com DAP maior que $35 \mathrm{~cm}$ não foram atacadas. Constataram, ainda, que quando o ataque de $S$. noctilio é mantido por vários anos no mesmo povoamento, a média do tamanho de árvores suscetíveis aumenta progressivamente.

No tratamento $\mathrm{T} 1$ notou-se que a infestação iniciou, na classe de $10 \mathrm{~cm}$ e nos demais tratamentos, com exceção do T2 da fazenda Goiabeira, o início se dá na classe de $15 \mathrm{~cm}$. Isto ocorreu porque nos tratamentos $\mathrm{T} 2$, T3, T4 e T5, o desbaste foi progressivamente mais pesado, acarretando em um número inferior de árvores remanescentes com diâmetros menores.

Quadro 1: Evolução do DAP médio, nos tratamentos das Fazendas Cerro Pelado, Goiabeira e Marco, Santa Catarina

Table 1: Evolution of average DBH in Cerro Pelado, Goiabeira and Marco Farms, Santa Catarina

\begin{tabular}{|c|c|c|c|c|c|c|c|}
\hline \multirow{2}{*}{ Local } & \multirow{2}{*}{ Tratamento } & \multicolumn{6}{|c|}{ DAP médio $(\mathrm{cm})$} \\
\cline { 3 - 7 } Cerro Pelado & & 92 & 93 & 94 & 95 & 96 & 99 \\
\hline & T1 & 22,4 & 23,1 & 23,4 & 23,8 & 24,4 & 24,5 \\
& T2 & 22,0 & 22,4 & 23,6 & 23,9 & 24,3 & 25,5 \\
& T3 & 21,4 & 21,9 & 23,6 & 24,1 & 24,8 & 26,1 \\
& T4 & 21,4 & 21,9 & 23,6 & 24,1 & 24,9 & 26,3 \\
& T5 & 21,4 & 21,8 & 24,5 & 25,0 & 26,0 & 27,6 \\
\hline \hline \multirow{5}{*}{ Goiabeirayyyyyyy} & T1 & & 20,5 & 21,2 & 21,5 & 22,1 & 22,6 \\
& T2 & & 21,3 & 23,0 & 23,6 & 24,7 & 25,5 \\
& T3 & & 20,9 & 22,5 & 23,2 & 24,3 & 25,2 \\
& T4 & & 21,1 & 23,6 & 24,3 & 25,7 & 27,1 \\
& T5 & & 21,3 & 24,1 & 25,0 & 26,5 & 27,9 \\
\hline \hline \multirow{5}{*}{ Marco } & T1 & 20,5 & 21,1 & 21,9 & 22,3 & 23,2 & 23,9 \\
& T2 & 19,9 & 20,7 & 21,8 & 22,7 & 24,0 & 25,2 \\
& T3 & 20,0 & 20,7 & 23,0 & 23,7 & 24,9 & 26,1 \\
& T4 & 20,5 & 21,1 & 23,7 & 24,5 & 25,8 & 27,1 \\
& T5 & 20,9 & 21,5 & 24,2 & 25,2 & 26,6 & 28,1 \\
\hline
\end{tabular}


Evolução da infestação por Sirex noctilio...

Figura 1: Evolução do DAP médio aritmético nos diferentes tratamentos das Fazendas Cerro Pelado, Goiabeira e Marco, Santa Catarina

Figure 1: Evolution of average DBH in Cerro Pelado, Goiabeira e Marco Farms, Santa Catarina
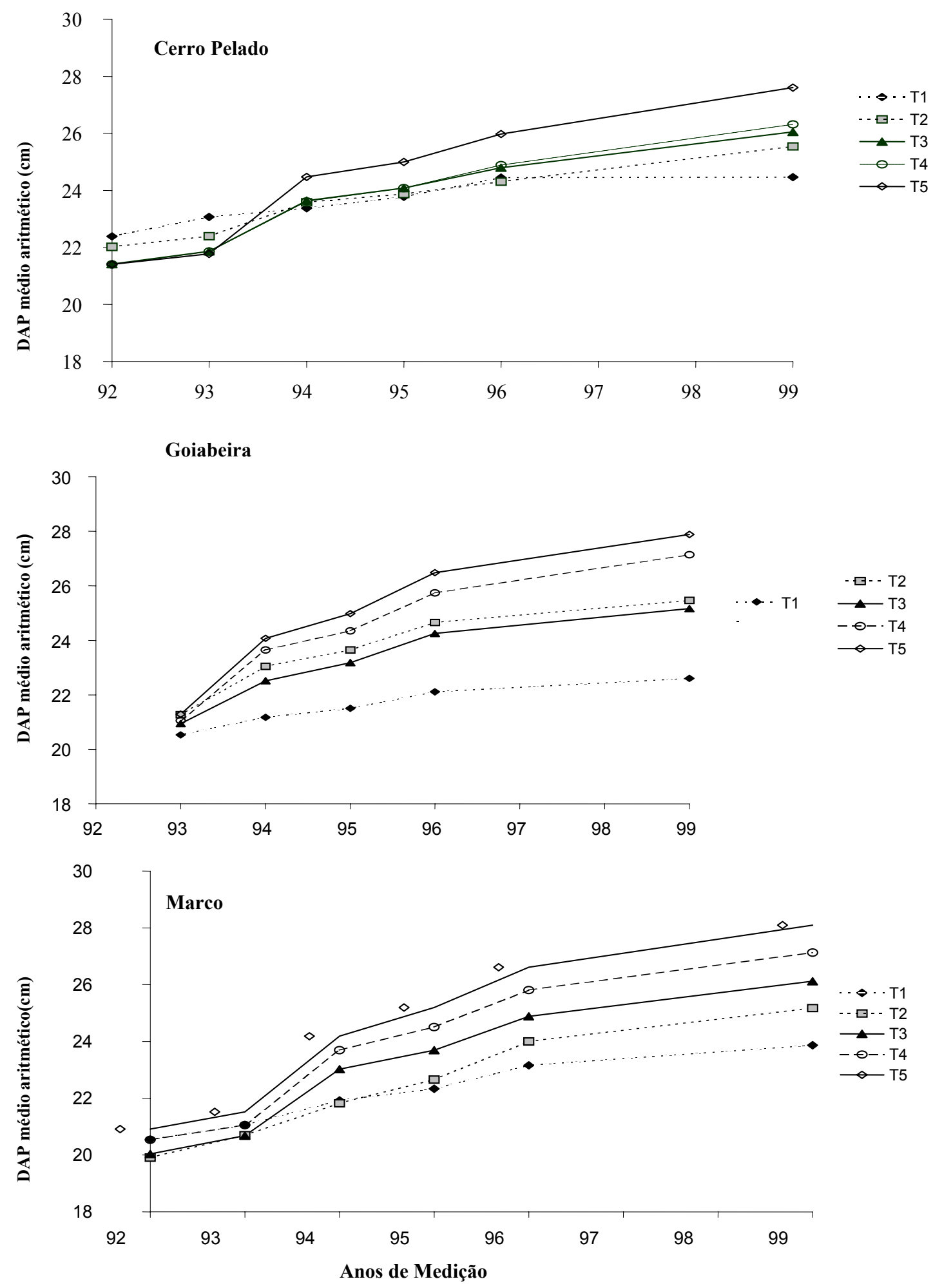
Figura 2: Distribuição das árvores atacadas por Sirex noctilio por tratamento e classes de DAP, nas Fazendas Cerro Pelado, Santa Catarina

Figure 2: Distribution of trees attacked by Sirex noctilio according to treatments, DBH classes in Cerro Pelado Farm, Santa Catarina
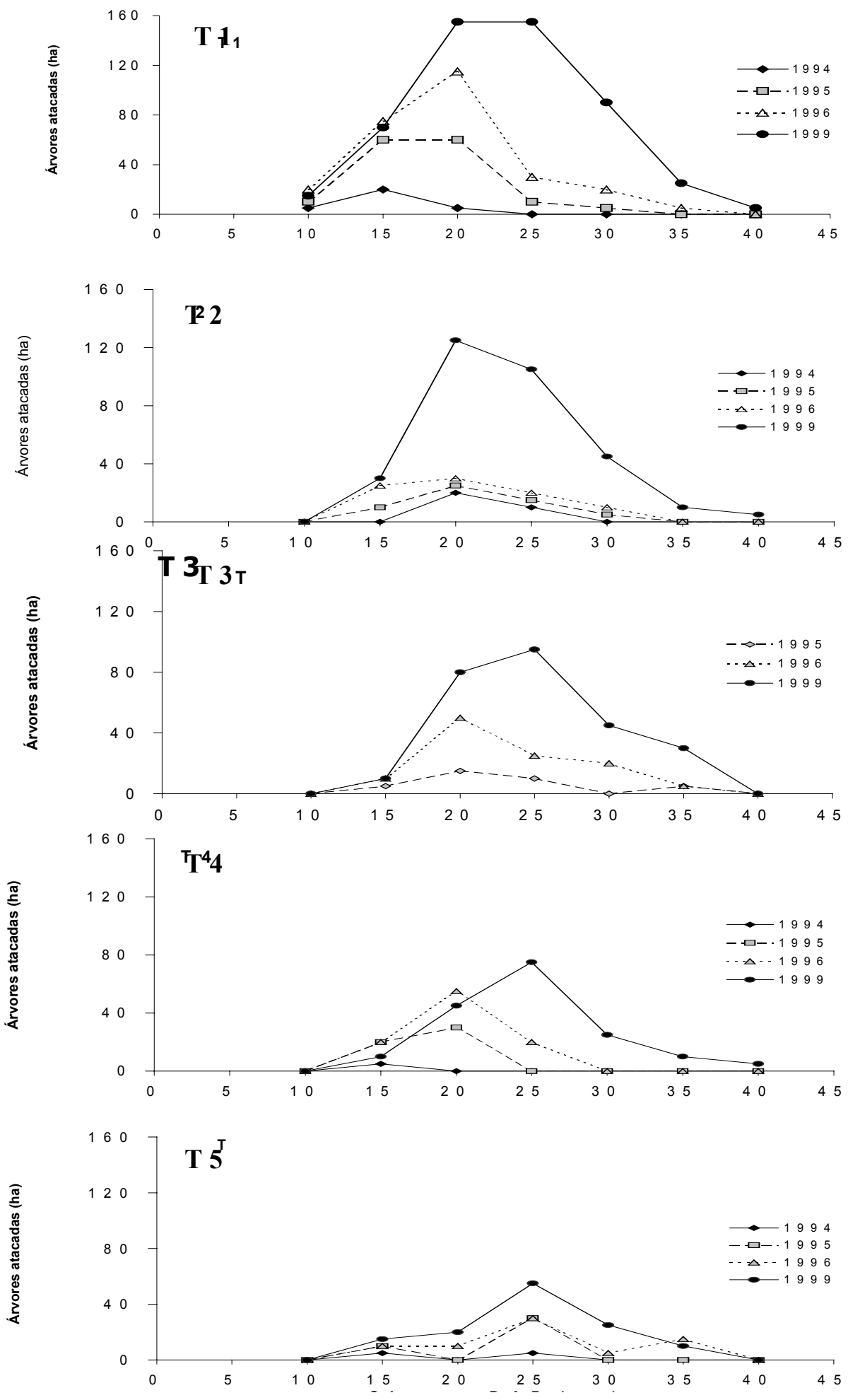

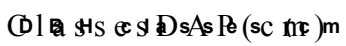


Evolução da infestação por Sirex noctilio...

Figura 3: Distribuição das árvores atacadas por Sirex noctilio por tratamento e classes de DAP, na Fazendas Goiabeira, Santa Catarina

Figure 3: Distribution of trees attacked by Sirex noctilio according to treatments, DBH classes in Goiabeira Farm, Santa Catarina
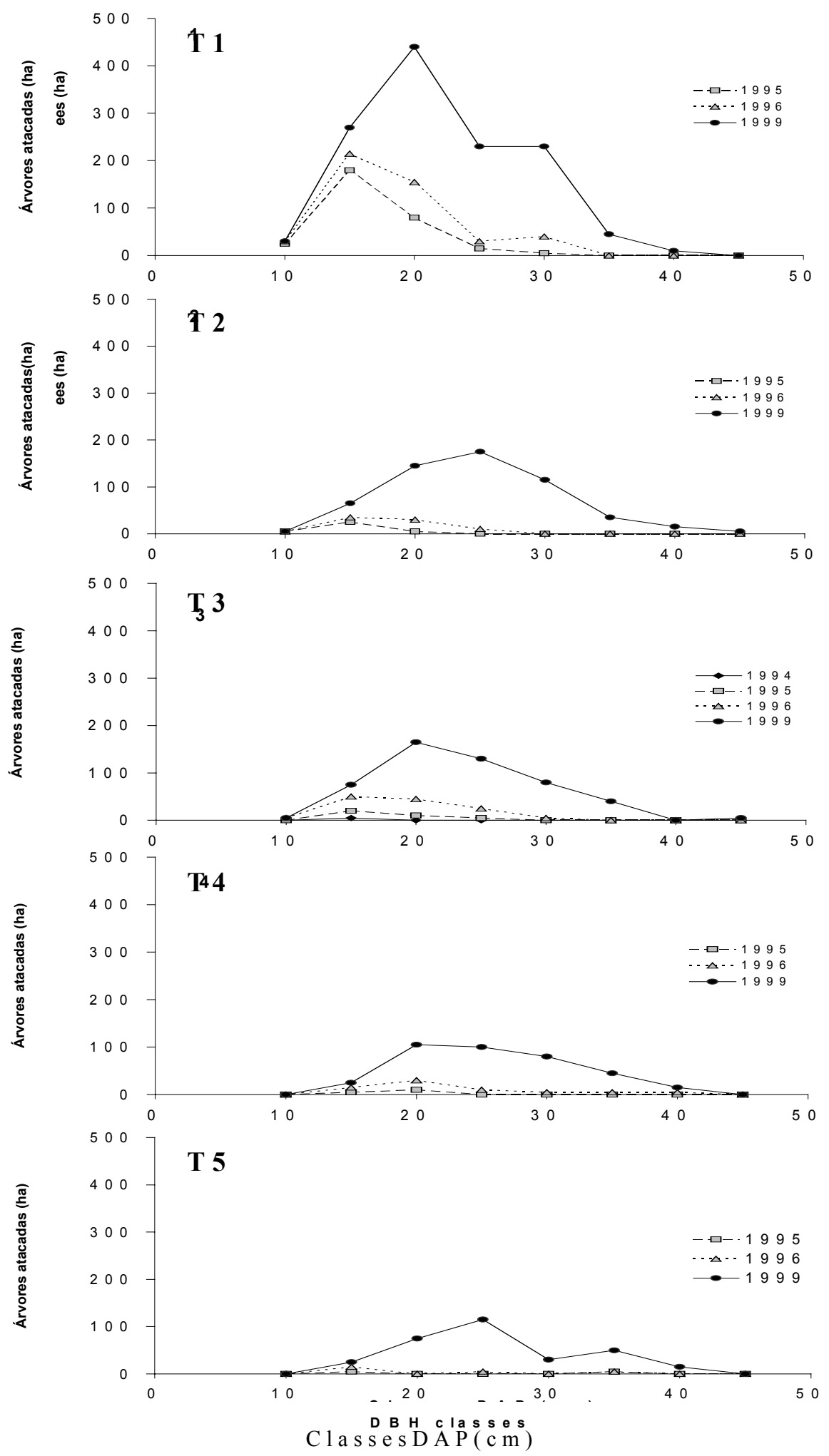
Figura 4: Distribuição das árvores atacadas por Sirex noctilio por tratamento e classes de DAP, na Fazendas Marco, Santa Catarina

Figure 4: Distribution of trees attacked by Sirex noctilio according to treatments, DBH classes in Marco Farm, Santa Catarina
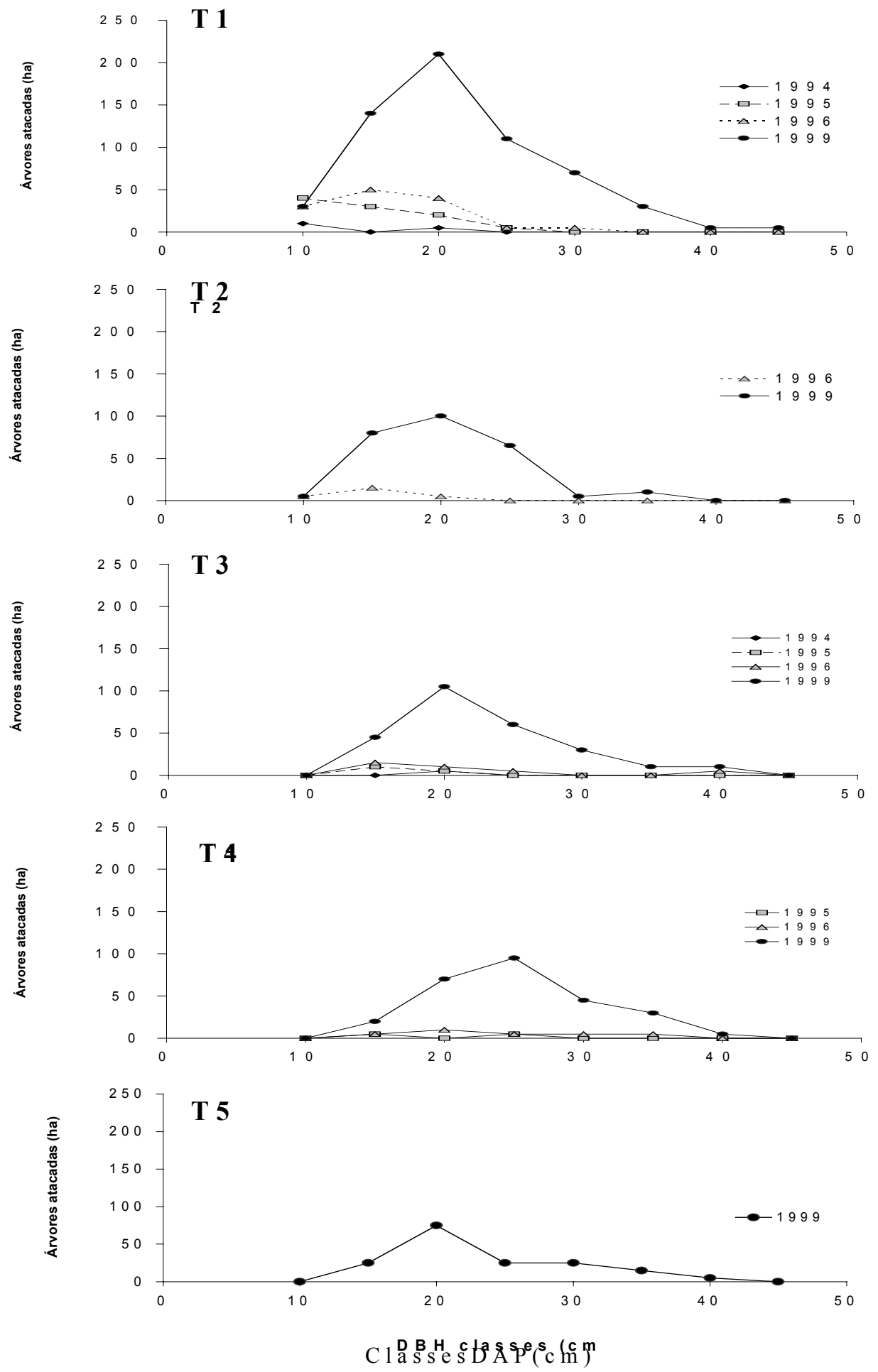


\section{CONCLUSÕES}

Neste estudo, verificou-se que o ataque da vespa-da-madeira tem inicio nas árvores de menor diâmetro, atingindo as de maior diâmetro com a evolução da infestação, ocorrendo um deslocamento ascendente do pico de ataque das classes de menor para as de maior diâmetro. Verificou-se, ainda, que o desbaste reduziu a infestação da vespa-damadeira, principalmente nos anos iniciais de infestação, e retardou sua evolução.

\section{REFERÊNCIAS}

ALFARO, R. I. Role of genetic resistance in management ecosystems susceptible to white pine weevil. Forestry Chronicle. Quebec: v. 72, n. 4, p. 374-380, 1996.

COUTTS, M. P. Rapid physiological change in Pinus radiata following attack by Sirex noctilio and its associated fungus Amylostereum spp. Australian Journal of Biological Science. Victoria: v. 30, n. 7, p. 274-276, 1968.

DAVIS, K. P. Forest management: regulation and valuation. New York: Mc Graw-Hill Book Company. 1966. 519 p.

IEDE, E. T.; PENTEADO, S. R. C.; BISOL, J. C. Primeiro registro de ataque de Sirex noctilio em Pinus taeda no Brasil. Curitiba: EMBRAPA-CNPF. 1988. 12 p. (Circular Técnica).

MADDEN, J. L. An analysis of an outbreak of the woodwasp Sirex noctilio F. (Hymenoptera: Siricidae), in Pinus radiata. Bulletin of Entomological Reseach. London: v. 65, p. 491-500, 1975.
MAINARDI, G. L.; SCHNEIDER, P. R.; FINGER, C. A. G. Produção de Pinus taeda na região de Cambará do Sul, RS. Revista Ciência Florestal. Santa Maria: v. 6, n. 1, p 39-52, nov. 1996.

MITCHELL, R. G.; WARNING, R. H.; PITMAN, G. B. Thinning lodgepole pine increases tree vigour and resistance to mountain pine beetle. Forest Science. Washington: v. 29, p. 204-221. 1983.

NEUMANN, F. G. Insect pest management in Australian radiata pine plantations. Australian Forestry, Canberra: v. 42, p. 30-38, 1979.

NEUMANN, F. G.; MOREY, J. L.; McKIMM, R. J. The Sirex wasp in Victoria. Land and Forest Division Bulletin. Victoria: v. 29, p. 141, 1987.

SCOLFORO, J. R. S.; MAESTRI, R. O manejo de florestas plantadas. In: SCOLFORO, J. R. S. Manejo Florestal. Lavras: UFLA/FAEPE. 1997. p. 315-380.

SMITH, D. M. The practice of silviculture. 8 Ed. New York: John Wiley \& Sons. 1986. 527 p.

TAYLOR, K. L. The Sirex woodwasp: ecology and control of introduced forest insect. In: The Ecology of Pests. Some Australian case histories. CSIRO Australia: 1981. p. 231248.

WATERS, W. E.; STARK, R. W. Forest Pest Management: Concept and Reality. Annual Review Entomology. Palo Alto: v. 25, p. 479509, 1980. 